\title{
Comparative Molecular Dynamics and Monte Carlo Study of Statistical Properties for Coarse-Grained Heteropolymers
}

\author{
JAKOB SCHLUTTIG, ${ }^{1,2}$ MICHAEL BACHMANN, ${ }^{1}$ WOLFHARD JANKE ${ }^{1}$ \\ ${ }^{1}$ Institut für Theoretische Physik and Centre for Theoretical Sciences (NTZ), Universität Leipzig, \\ Postfach 100 920, D-04009 Leipzig, Germany \\ ${ }^{2}$ Center for Modelling and Simulation in the Biosciences (BIOMS), Ruprecht-Karls-Universität Heidelberg, \\ Im Neuenheimer Feld 267 (BIOQUANT), D-69120 Heidelberg, Germany \\ Received 28 November 2007; Accepted 7 March 2008 \\ DOI 10.1002/jcc.21003 \\ Published online 13 May 2008 in Wiley InterScience (www.interscience.wiley.com).
}

\begin{abstract}
Employing a simple hydrophobic-polar heteropolymer model, we compare thermodynamic quantities obtained from Andersen and Nosé-Hoover molecular dynamics as well as replica-exchange Monte Carlo methods. We find qualitative correspondence in the results, but serious quantitative differences using the Nosé-Hoover chain thermostat. For analyzing the deviations, we study different parameterizations of the Nosé-Hoover chain thermostat. Autocorrelations from molecular dynamics and Metropolis Monte Carlo runs are also investigated.
\end{abstract}

(C) 2008 Wiley Periodicals, Inc. J Comput Chem 29: 2603-2612, 2008

Key words: molecular dynamics; Nosé-Hoover thermostat; Andersen thermostat; replica-exchange Monte Carlo; coarse-grained heteropolymers

\section{Introduction}

Understanding protein folding is one of the most complex challenges of science. The main reason is the characteristic property common to all proteins that the individual biological function strongly correlates with the three-dimensional geometry of the native fold. Proteins are functional ingredients in all biological systems and misfolding, mutational, or nonfunctional aggregation typically entail influential disturbances in biological networks which frequently lead to still incurable diseases. For this reason, there is an enormously growing interdisciplinary interest in understanding the often spontaneous structure formation of proteins, which generally depends on the linear sequence of amino acids building up the macromolecule. Most of the proteins consist of hundreds of amino acids and, therefore, thousands of atoms. The folding process is guided by usual noncovalent physical and chemical interactions, such as Coulomb forces due to residual charges, hydrogen bonding, submolecular van der Waals potentials, energetic torsional barriers, and the effective hydrophobic force, which results as an effective interaction with the aqueous solvent surrounding most of the non-membrane proteins.

Beside the extensive bioanalytical efforts in the past few decades to resolve by X-ray and NMR techniques native folds of tens of thousands of bioproteins, computational simulation methods have become very popular tools for studying experimentally hardly manageable dynamical and thermodynamic properties accompanying the folding kinetics. In particular, this incorporates the central question of the cooperative conformational transitions the protein experiences in the formation of secondary structures (e.g., helices, sheets) and tertiary folds (e.g., hydrophobic-core domains).

In computational chemistry and physics, three main simulation techniques are used, which primarily focus on different aspects. Dynamical quantities of a process are frequently addressed by means of molecular dynamics (MD) methods, ${ }^{1,2}$ where the phase-space trajectory of a system is numerically calculated employing a discrete, but symplectic integration scheme for the system's equations of motion. One of the essential properties of Hamiltonian dynamics in continuum is that the phase-space density is constant in time. Thus, a necessary condition for a discrete symplectic integration is that the Jacobian of the transformation from the system's state at time $t$ to the state at time $t+\Delta t$ is unity. Conversely, Monte Carlo (MC) simulations are typically used to reveal the thermodynamic equilibrium

Correspondence to: M. Bachmann; e-mail: Michael.Bachmann@itp.unileipzig.de

Contract/grant sponsor: DFG (German Science Foundation); contract/grant number: JA 483/24-1

Contract/grant sponsors: John von Neumann Institute for Computing (NIC), Forschungszentrum Jülich; contract/grant number: hlz11 
properties of a system by sampling system conformations in the thermally relevant region of the phase space. ${ }^{3,4}$ Since configurational updates in MC are in fact based on a Markov process, MC also possesses a kind of pseudo-dynamics. The third frequently used method is based on the density functional and therefore provides a tool for ab-initio quantum-chemical calculations of electronic structures of many-particle systems. ${ }^{5}$

From a statistical physics point of view, conventional MD samples a microcanonical ensemble, ${ }^{6}$ as the system energy is kept constant. However, thermal fluctuations in the physiological temperature interval are of essential importance for biological processes. Therefore, in more realistic simulations, the energy should fluctuate according to a certain, e.g., Boltzmannian distribution. In consequence, the system's degrees of freedom have to be coupled to an environment, e.g., a heat-bath to provide constant temperature. In our MD simulations, we used the stochastic Andersen thermostat ${ }^{2,7}$ and the deterministic Nosé-Hoover chain (NHC) thermostat. $^{2,8-10}$ It was shown for polymer systems that ensemble averages of dynamic properties are unaffected by coupling Newtonian dynamics with Nosé-Hoover thermostats. ${ }^{11,12}$ Thermostats were introduced to ensure that the system trajectory in principle samples the phase space according to the Boltzmann distribution. This means that canonical statistics should be exactly satisfied in an infinitely long thermostated MD run. In practice, however, the question is what is a sufficiently long run to accumulate reliable statistics. By definition, MD generates an extremely local "update" within a single time step, whereas MC sweeps provide larger jumps in the phase space. Therefore, it is to be expected that correlations decay much slower in MD, compared to MC.

In this paper, we aim at a comparative study of thermostated $\mathrm{MD}^{2,7-10}$ and replica-exchange MC. ${ }^{13}$ To this end, we consider a simplified coarse-grained bead-spring heteropolymer model and concentrate on thermodynamic properties. The paper is organized as follows. In Models and Methods, the AB model, modified by flexible virtual bonds, is revisited. Furthermore, we summarize the essentials of the Andersen and the NHC thermostat used in our constant-temperature MD simulations, and the replica-exchange generalized-ensemble MC method which is used for generating reference data. The results obtained with these methods are discussed and the deviations are analysed in Thermodynamics of S1: Comparison of Results from MD and MC Simulations. The paper is concluded by a summary.

\section{Model and Methods}

In this section, we introduce the model employed in our comparative study and summarize the details of the methods used.

\section{Hydrophobic-Polar Heteropolymer with Flexible Covalent Bonds}

Our study is based on a simple coarse-grained heteropolymer model, which is strongly related to the $\mathrm{AB}$ model,${ }^{14}$ where only hydrophobic $(A)$ and hydrophilic $(B)$ monomers are distinguished. In the original $\mathrm{AB}$ model, covalent bonds between adjacent monomers have a fixed length. This corresponds to the known stiffness of these virtual bonds. In fact, a "covalent" bond in the AB model between successive monomers is a virtual bond as it actually corresponds to the distance of the $\mathrm{C}^{\alpha}$ atoms of adjacent amino acids. This virtual bond comprises three covalent bonds $\left(\mathrm{C}_{n}^{\alpha}-\mathrm{C}_{n}^{\prime}-\mathrm{N}_{n+1}-\mathrm{C}_{n+1}^{\alpha}\right)$ and its length is nearly constant in real peptides $(\approx 3.8 \AA)$. Here, we relax this constraint through replacing the stiff bond by a harmonic spring. The reason is of rather technical nature as it drastically simplifies the MD implementation using Cartesian coordinates. MD simulations of polymers with stiff bonds are in principle possible, e.g., by using the standard SHAKE ${ }^{15}$ or RATTLE ${ }^{16}$ algorithms.

Denoting the set of coordinates for the $N$ monomers by $\mathbf{R}=$ $\left\{\mathbf{r}_{1}, \ldots, \mathbf{r}_{N}\right\}$, we define our bead-spring variant of the $\mathrm{AB}$ model with $\varepsilon_{0}$ being an overall energy scale as

$$
V(\mathbf{R})=\varepsilon_{0}\left[v_{\text {bend }}(\mathbf{R})+v_{\mathrm{LJ}}(\mathbf{R})+v_{\text {harm }}(\mathbf{R})\right],
$$

where

$$
v_{\text {bend }}=\frac{1}{4} \sum_{k=1}^{N-2}\left(1-\cos \vartheta_{k}\right)
$$

is the bending energy and the sum runs over the $(N-2)$ bending angles of successive bond vectors. The monomer-type dependent intramolecular potential between nonbonded monomers $i$ and $j$ with distance $r_{i j}=\left|\mathbf{r}_{i}-\mathbf{r}_{j}\right|$ is of Lennard-Jones form:

$$
v_{\mathrm{LJ}}=4 \sum_{i=1}^{N-2} \sum_{j=i+2}^{N}\left(\frac{1}{r_{i j}^{12}}-\frac{C\left(\sigma_{i}, \sigma_{j}\right)}{r_{i j}^{6}}\right) .
$$

The monomer-type dependence of this contribution is expressed by the parameter $C$ :

$$
C\left(\sigma_{i}, \sigma_{j}\right)= \begin{cases}+1, & \sigma_{i}, \sigma_{j}=A, \\ +1 / 2, & \sigma_{i}, \sigma_{j}=B, \\ -1 / 2, & \sigma_{i} \neq \sigma_{j} .\end{cases}
$$

The third term in eq. (1) is the harmonic-spring extension of the $A B$ model and reads:

$$
v_{\text {harm }}=\alpha \sum_{l=1}^{N-1}\left(r_{l l+1}-b_{0}\right)^{2} .
$$

The sum is taken over the $N-1$ bonds and therefore the spring energy is related to the square deviation of the bond length from the minimum-potential distance $b_{0}$, which sets the characteristic length scale. The parameter $\alpha$ controls the bond strength and in the strongcoupling limit $\alpha \rightarrow \infty$, the fixed-bond behavior of the original $\mathrm{AB}$ model is approached.

The kinetic energy is $E_{\text {kin }}(\mathbf{P})=\mathbf{P}^{T} \mathbf{P} / 2 m$, where $\mathbf{P}=$ $\left\{\mathbf{p}_{1}, \ldots, \mathbf{p}_{N}\right\}$ is the set of the $N$ monomer momentum vectors. Independent of their type, all monomers shall have the same mass $m$ of the order of the average mass of an amino acid. The Hamiltonian

$$
\mathcal{H}(\mathbf{P}, \mathbf{R})=E_{\text {kin }}(\mathbf{P})+V(\mathbf{R})
$$


is equivalent to the total energy of the system and constant in time, $\mathcal{H}(\mathbf{P}, \mathbf{R}) \equiv E=$ const. Throughout the heteropolymer simulations, natural units are employed, in which $\varepsilon_{0}=b_{0}=m=k_{B}=1$.

In this comparative study, we concentrate ourselves on the exemplified $\mathrm{AB}$ heteropolymer sequence $\mathrm{S} 1: B A_{2} B A_{4} B A B A_{2} B A_{5} B$ with 20 monomers [14 being hydrophobic $(A)$ and 6 polar $(B)]$. The thermodynamic properties of the fixed-bond heteropolymer with this sequence have been analysed in detail in Ref. 17. The AB model with fixed bond lengths has also proven quite useful in a systematic characterisation of heteropolymer folding channels known from realistic proteins. ${ }^{18}$

\section{Molecular Dynamics with Andersen and Nosé-Hoover Thermostat}

Conventional molecular dynamics is governed by Newton's equations of motion, which read in Hamiltonian form for the $i$ th particle

$$
\begin{aligned}
\dot{\mathbf{r}}_{i} & =\nabla_{\mathbf{p}_{i}} \mathcal{H}(\mathbf{P}, \mathbf{R})=\frac{\mathbf{p}_{i}}{m}, \\
\dot{\mathbf{p}}_{i} & =-\nabla_{\mathbf{r}_{i}} \mathcal{H}(\mathbf{P}, \mathbf{R})=-\nabla_{\mathbf{r}_{i}} V(\mathbf{R}) .
\end{aligned}
$$

System trajectories lie on a constant-energy surface, $E=$ const. To conserve the energy in molecular dynamics simulations with discretized time steps, symplectic integrators are required, which provide the stability of the phase-space trajectory under time reversal.

In this standard form of molecular dynamics, the states of the system form a microcanonical ensemble in an energy shell $E-\Delta E<$ $E<E+\Delta E$ with $\Delta E / E \ll 1$. The temperature does not serve as an external control parameter and is defined as $T=(\partial S(E) / \partial E)^{-1}$ via the microcanonical entropy $S(E)=k_{B} \ln \int_{E_{\min }}^{E} d E^{\prime} g\left(E^{\prime}\right)$, where $g(E)$ is the density of states with energy $E .^{19}$

In many applications, however, it is desirable to adjust the system temperature $T$ by a heat bath. Consequently, the total energies of system states follow the canonical Boltzmann distribution $p_{\text {can }}(E) \sim g(E) \exp \left(-E / k_{B} T\right)$. Typically, the folding temperature of a peptide determines the characteristic energy scale. There are mainly two classes of approaches to introduce thermostats into Hamilton's equation of motion: by stochastic collision forces or via virtual deterministic extensions of the phase space. In our MD simulations, we have used the stochastic Andersen thermostat ${ }^{7}$ and the deterministic Nosé-Hoover chain. ${ }^{10}$

Using the Andersen thermostat, a monomer experiences a random collision with a fictitious heat-bath particle after each time step $\delta t$ with probability $p_{\text {coll }}=v \delta t$, where $v$ is the collision frequency. Therefore, for uncorrelated random forces, the probability for a collision at time $t$ is Poissonian, $p_{\text {coll }}(v, t)=v \exp (-v t)$. In case of a collision, each component of the new momentum $p=p_{i}^{(j)}, j=1,2,3$, of the selected monomer is drawn from the canonical Maxwell-Boltzmann distribution $p_{\text {can }}^{\text {kin }}(p)=$ $\exp \left(-p^{2} / 2 m k_{B} T\right) / \sqrt{2 \pi m k_{B} T}$, whose width is determined by the temperature $T$. In Andersen dynamics, each monomer behaves like a Brownian particle under the influence of the external field induced by the other nonbonded monomers and the springs. After infinitely long time $t$, the phase-space trajectory, consisting of a set of nonconnected deterministic fragments, will have covered the complete accessible phase space, which is sampled according to the Boltzmann distribution $p_{\text {can }}(E)$.

Nosé-Hoover dynamics is more complex, as the phase space is extended by $2 M$ additional degrees of freedom, $\xi_{k}$ and $p_{\xi_{k}}$, $k=1, \ldots, M$. These dynamical variables effectively represent the coupling of the system to the heat bath. The idea is that the highdimensional phase space provides the particles with more flexibility to leave the $E=$ const. trajectory in the "true" $(\mathbf{P}, \mathbf{R})$ phase space in a completely deterministic "extended" dynamics. The Nosé-Hoover energy

$$
H_{\mathrm{NHC}}=\mathcal{H}(\mathbf{P}, \mathbf{R})+\sum_{k=1}^{M} \frac{p_{\xi_{k}}^{2}}{2 Q_{k}}+3 N k_{B} T \xi_{1}+k_{B} T \sum_{k=2}^{M} \xi_{k}
$$

which is conserved in the multi-dimensional phase space, $H_{\mathrm{NHC}}=$ const., is defined in such a way that by integrating out the fluctuations of the additional degrees of freedom, the $(\mathbf{P}, \mathbf{R})$ states are distributed according to the canonical ensemble. However, the extended system is not Hamiltonian anymore and the derivation of the equations of motion requires some care. Extending the Hamiltonian equations of motion (7) and (8), the Nosé-Hoover equations of motion $\operatorname{read}^{2,8}$ :

$$
\begin{aligned}
\dot{\mathbf{r}}_{i} & =\frac{\mathbf{p}_{i}}{m}, \\
\dot{\mathbf{p}}_{i} & =-\nabla_{\mathbf{r}_{i}} V(\mathbf{R})-\frac{p_{\xi_{1}}}{Q_{1}} \mathbf{p}_{i}, \\
\dot{\xi}_{k} & =\frac{p_{\xi_{k}}}{Q_{k}}, \\
\dot{p}_{\xi_{1}} & =\left(\sum_{i=1}^{N} \frac{\mathbf{p}_{i}^{2}}{m}-3 N k_{B} T\right)-\frac{p_{\xi_{2}}}{Q_{2}} p_{\xi_{1}}, \\
\dot{p}_{\xi_{k}} & =\frac{p_{\xi_{k-1}}^{2}}{Q_{k-1}}-k_{B} T-\frac{p_{\xi_{k+1}}}{Q_{k+1}} p_{\xi_{k}}\left(1-\delta_{k M}\right) .
\end{aligned}
$$

The numerical values of the virtual masses $Q_{k}$ of the coupling variables influence the dynamics but, in principle, not the statistical averages. For systems, where the total energy is the only conserved quantity in the dynamics, the choice of a single coupling coordinate $M=1$ is sufficient. To destroy additional symmetries, however, $M>1$ couplings are required and their Nosé-Hoover equations of motion form the linear Nosé-Hoover chain. ${ }^{2,10}$ A prominent exceptional example is the one-dimensional harmonic oscillator, where two coupling degrees of freedom are necessary.

The numerical integration of the equations of motion in our simulations with Andersen and Nosé-Hoover thermostat was performed with the standard Störmer-Verlet algorithm. ${ }^{2,20}$

\section{Implementation Details of the NHC Thermostat}

In Nosé-Hoover dynamics, the temporal propagation for a time step $\delta t$ of the system and the heat-bath coupling degrees of freedom is governed by the time evolution operator $U_{\mathrm{NHC}}(\delta t)$, which can be 
decomposed into the Trotter factorized form

$$
\begin{aligned}
U_{\mathrm{NHC}}(\delta t)= & e^{i \mathcal{L}_{C} \delta t / 2} e^{i \mathcal{L}_{R} \delta t / 2} \\
& \times e^{i \mathcal{L}_{P} \delta t} e^{i \mathcal{L}_{R} \delta t / 2} e^{i \mathcal{L}_{C} \delta t / 2}+\mathcal{O}\left(\delta t^{3}\right) .
\end{aligned}
$$

In this expression, $\mathcal{L}_{P}, \mathcal{L}_{R}$, and $\mathcal{L}_{C}$ are the Liouville operators of the monomer momenta $\mathbf{P}$, the monomer coordinates $\mathbf{R}$, and the heat-bath coupling chain degrees of freedom, respectively. In higherorder integration schemes, the time step of heat-bath coupling propagation is divided further into $n_{c}$ equidistant steps. Thus,

$$
e^{i \mathcal{L}_{C} \delta t / 2}=\prod_{j=1}^{n_{c}} \prod_{k=1}^{m} e^{i \mathcal{L}_{C} w_{k} \delta t / 2 n_{c}} .
$$

In our NHC-MD simulations, we mainly followed the procedure described in Ref. 21. We applied a 5th order integration scheme ( $m=3$ ) and set $n_{c}=1$, i.e., the error is of order $\mathcal{O}\left(\delta t^{5}\right)$. The Yoshida-Suzuki parameters $w_{k}$ are $w_{1,3}=1 /\left(2-2^{1 / 3}\right), w_{2}=1-$ $2 w_{1} \cdot{ }^{22,23}$

\section{The Choice of the Virtual Masses for the Heat-Bath-Coupling Degrees of Freedom}

The masses $Q_{k}(k=1, \ldots, M)$ of the virtual heat-bath "particles" influence the coupling strength and, therefore, the dynamics of the correlations between the degrees of freedom of the heat-bath and the system. Large thermal inertia $Q_{k}$ cause a large time scale for the fluctuations of the heat-bath degrees of freedom. Depending on the fastest time scale of the system, the thermostat may not be capable in balancing these fluctuations. On the other hand, too small values of $Q_{k}$ induce high-frequency fluctuations into the system, which equilibrates then very slowly.

For the one-dimensional harmonic oscillator $\mathcal{H}=p^{2} / 2 m+$ $m \omega^{2} x^{2} / 2$ with $m=2$ and $\omega^{2}=1 / 2$, Figure 1 shows for different choices of $Q_{1}$ and $Q_{2}$ the relative errors of the canonical position and momentum distributions as measured in NHC-MD simulations with $M=2$ Nosé-Hoover thermostats, compared with the exact distributions. The data were obtained by performing $10^{7}$ time steps of width $\delta t=0.01$ at $T=5$. The relative error of the measured histogram compared with the exact distribution is noticeably dependent on the values of $Q_{1}$ and $Q_{2}$. The biggest errors are found for very small and very large $Q$-values, whereas fluctuations and response seem to be balanced much better for moderate choices of order $Q_{1,2} \sim \mathcal{O}(1)$. This qualitatively confirms the suggestion in Ref. 10 to relate the $Q_{k}$ 's to the fastest time scales of the heat-bath (as induced by the thermal energy $\left.\sim k_{B} T\right)$ and the system $(\tau=1 / \omega)$ by choosing

$$
Q_{k}=f_{k} k_{B} T \tau^{2},
$$

where $f_{1}=D N(D$ is the spatial dimension $)$ and $f_{k>1}=1$. As we can also infer from Figure 1, our NHC-MD implementation works quite well for the one-dimensional harmonic oscillator with the properly adjusted virtual masses $\left(Q_{1}=Q_{2}=10\right.$ in our units).

For more complex systems, the identification of $\tau$ is not obvious. It can be related, e.g., to the fastest fluctuations and thus the largest mode in the spectrum of autocorrelation functions. The normalized autocorrelation function of a time-dependent quantity $s(t)$ is defined as

$$
A_{s}(\Delta t)=\frac{\langle s(t) s(t+\Delta t)\rangle-\langle s(t)\rangle^{2}}{\left\langle s(t)^{2}\right\rangle-\langle s(t)\rangle^{2}}
$$

where $\langle\ldots\rangle$ is the temporal average over the time series, which in equilibrium is identical with the statistical ensemble average.

In Figure 2, the Fourier transforms, i.e., the frequency spectra, of the velocity autocorrelation function, $\tilde{A}_{v}(\omega)$, and of the bondlength autocorrelations, $\tilde{A}_{r_{i i+1}}(\omega)$, are shown for the heteropolymer sequence $\mathrm{S} 1$. Assuming that the fluctuations of the harmonic springs (5) are of shortest time scale, the bond strength $\alpha=m \omega_{\text {bond }}^{2} / 2$ defines the time scale:

$$
\tau_{\text {bond }}=\omega_{\text {bond }}^{-1}=\sqrt{\frac{m}{2 \alpha}} .
$$

The results for the autocorrelations obtained with an exemplified NHC-MD run at $T=1.0$ with $M=2$ Nosé-Hoover thermostats as shown in Figure 2 justify this assumption. Both autocorrelation functions have a peak close to $\omega / \omega_{\text {bond }}=1$, which is the highestfrequency mode. Therefore, we use $\tau_{\text {bond }}$ for adjusting the virtual masses in eq. (17) in our NHC-MD heteropolymer simulations.

\section{Replica-Exchange Monte Carlo Method}

For verifying the statistical results obtained with NHC-MD of the heteropolymer model, a comparison with exact results is not possible. Therefore, we use a standard Markov chain Monte Carlo algorithm as reference method. Since the dynamics of conventional Metropolis sampling at fixed temperature notoriously slows down close to temperatures, where conformational transitions occur and also, in the dense polymer limit, a generalized-ensemble method is more appropriate for a comprising study of thermodynamics. A simple and efficient sampling scheme is provided by the replicaexchange or parallel tempering method. ${ }^{13}$ In this method, threads of Markov chains at different, deliberately chosen temperatures run in parallel and frequent trials to exchange conformations between the threads ensure a reasonable sampling of the accessible conformational space. In most applications, MC methods serve to accumulate statistics by sampling regions of the configurational space that dominate the ensemble at a given temperature. Although sometimes also employed for kinetic studies, the MC dynamics of the system is typically of less importance. For most quantities of interest, the kinetic part in the Hamiltonian (6) does not influence statistical averages, i.e., the momentum fluctuations can be integrated out exactly. Therefore, in our MC simulations, the system experiences only coordinate updates. In a replica-exchange step, the actual conformation $\mathbf{R}$ with reciprocal thermal energy $\beta=1 / k_{B} T$ is tried to be exchanged with the polymer conformation $\mathbf{R}^{\prime}$ being currently present in a neighbor thread running at $\beta^{\prime}=1 / k_{B} T^{\prime}$. The acceptance probability for this exchange is simply given by

$$
w\left(\mathbf{R} \leftrightarrow \mathbf{R}^{\prime} ; \beta, \beta^{\prime}\right)=\min \left(1, e^{-\Delta}\right),
$$




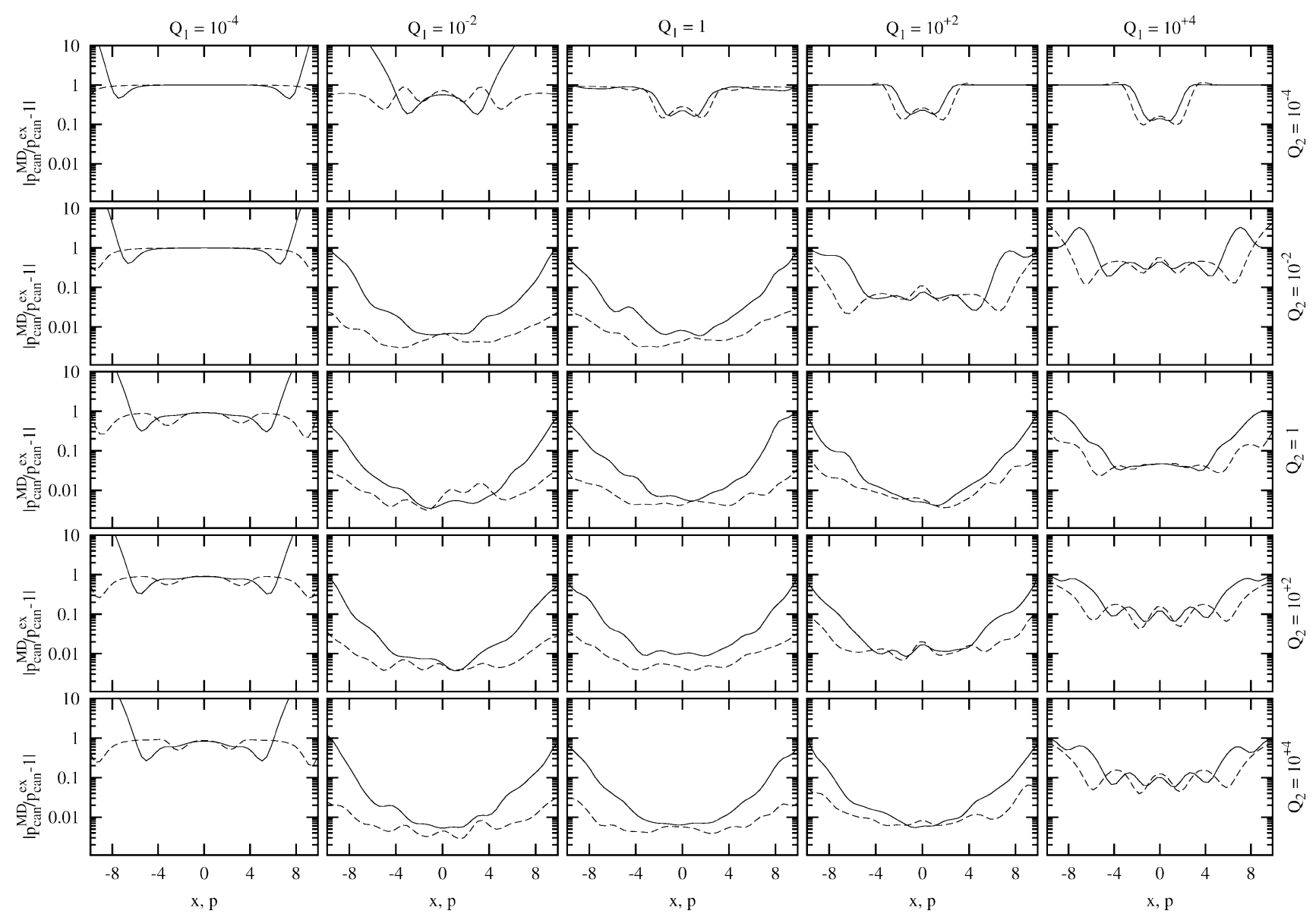

Figure 1. Relative errors $\left|p_{\mathrm{can}}^{\mathrm{MD}} / p_{\mathrm{can}}^{\mathrm{ex}}-1\right|$ of the canonical position (solid line) and momentum (dashed line) distributions $p_{\text {can }}(x)$ and $p_{\text {can }}(p)$ for a one-dimensional harmonic oscillator, estimated in NHC-MD simulations with $M=2$ for different choices of virtual masses $Q_{1}$ and $Q_{2}$ at $T=5$.

where $\Delta=\left(\beta^{\prime}-\beta\right)\left[V(\mathbf{R})-V\left(\mathbf{R}^{\prime}\right)\right]$. Hence, for $\Delta<0$, the exchange is always accepted. If $\Delta>0$, the exchange is accepted with the Boltzmann-like probability $e^{-\Delta}$. A reasonable acceptance rate can only be achieved, if the canonical histograms of the system have sufficient overlap at the exchange temperatures $T$ and $T^{\prime}$. Therefore, the efficiency of the method strongly depends on the careful choice of the number of threads and the associated temperatures. Conformational updates between the exchange trials within a thread at constant temperature are accepted according to the Metropolis transition probability. In fixed-bond simulations conformational changes were performed using spherical-cap updates ${ }^{17}$ For simulations of the spring model, we used simple Cartesian updates, where a monomer or a bond is moved. An enormous advantage of the method is that it can easily be parallelized as only the temperatures between the threads, running on individual processors, have to be communicated.

\section{Thermodynamics of S1: Comparison of Results from MD and MC Simulations}

In the following, we analyse the thermodynamic behavior of the flexible-bond model for the heteropolymer sequence S1

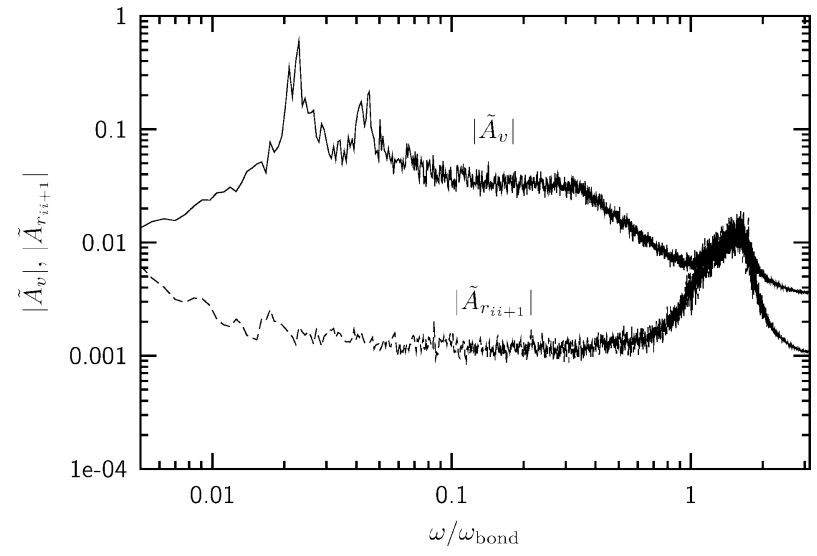

Figure 2. Frequency spectra of the velocity autocorrelation function $\tilde{A}_{v}(\omega)$ (upper, solid line) and of the bond-length autocorrelation $\tilde{A}_{r_{i i+1}}(\omega)$ (lower, dashed line) for bond strength $\alpha=50$ at $T=1$.

from results obtained with Andersen MD (A-MD), Nosé-Hoover chain MD (NHC-MD), standard Metropolis MC (M-MC), and replica-exchange MC (RE-MC) simulations. The methods 
Table 1. Methods and Specifications used in this Study.

\begin{tabular}{|c|c|c|c|c|}
\hline Method & Label & $M$ & Equilibration & Run length \\
\hline Metropolis MC & M-MC & & $1 \times 10^{7}$ & $1 \times 10^{8}$ \\
\hline Replica-Exchange MC & RE-MC & & $1 \times 10^{5}$ & $3 \times 10^{8}$ \\
\hline $\begin{array}{l}\text { MD with Andersen } \\
\text { thermostat }\end{array}$ & A-MD & & $1 \times 10^{8}$ & $3 \times 10^{8}$ \\
\hline \multirow{4}{*}{$\begin{array}{l}\text { MD with Nosé-Hoover } \\
\text { chain thermostat }\end{array}$} & 2NHC-MDs & 2 & $1 \times 10^{8}$ & $3 \times 10^{8}$ \\
\hline & 2NHC-MDl & 2 & $1 \times 10^{9}$ & $6 \times 10^{9}$ \\
\hline & 3NHC-MD & 3 & $1 \times 10^{9}$ & $6 \times 10^{9}$ \\
\hline & 4NHC-MD & 4 & $1 \times 10^{9}$ & $6 \times 10^{9}$ \\
\hline
\end{tabular}

Equilibration times and run lengths are given in MC sweeps or MD steps, respectively.

and their specifications in the simulations are listed in Table 1 .

\section{Energetic and Conformational Fluctuations}

Energetic fluctuations are expressed by the specific heat per monomer via $C_{V}(T)=\left(\left\langle\mathcal{H}^{2}\right\rangle-\langle\mathcal{H}\rangle^{2}\right) / N k_{B} T^{2}$. For Hamiltonian systems in three dimensions (6), this can be written as

$$
C_{V}(T)=C_{V}^{\mathrm{kin}}+\frac{1}{N k_{B} T^{2}}\left(\left\langle V(\mathbf{R})^{2}\right\rangle-\langle V(\mathbf{R})\rangle^{2}\right)
$$

with the constant kinetic contribution $C_{V}^{\text {kin }}=3 k_{B} / 2$. In our analyses, we consider only the potential energy contribution allowing for a direct comparison with results from MC simulations.

Frequently used conformational quantities in polymer physics are the end-to-end distance

$$
R_{\mathrm{ee}}(\mathbf{R})=\left|\mathbf{r}_{N}-\mathbf{r}_{1}\right|
$$

and the radius of gyration

$$
R_{\mathrm{gyr}}(\mathbf{R})=\sqrt{\frac{1}{N} \sum_{i=1}^{N}\left(\mathbf{r}_{i}-\mathbf{r}_{0}\right)^{2}}, \quad \mathbf{r}_{0}=\frac{1}{N} \sum_{i=1}^{N} \mathbf{r}_{i}
$$

which is a measure for the compactness of the polymer. In particular, the fluctuations

$$
\frac{\partial}{\partial T}\left\langle R_{\mathrm{ee}, \mathrm{gyr}}\right\rangle=\frac{1}{k_{B} T^{2}}\left(\left\langle R_{\mathrm{ee}, \mathrm{gyr}} E\right\rangle-\left\langle R_{\mathrm{ee}, \mathrm{gyr}}\right\rangle\langle E\rangle\right)
$$

are of interest, as divergences or extremal points in their temperature dependence are signals for cooperative conformational activity, i.e., they indicate conformational transitions. It should be noted that for finite-length systems, as protein like heteropolymers definitely are, the fluctuations do not collapse at a certain transition temperature. Rather, different quantities signalize activity typically at different temperatures forming a transition region. ${ }^{24}$

\section{Thermodynamic Properties of S1}

As a first application, we discuss how the thermodynamic behavior of the heteropolymer with sequence S1 depends on the flexibility of the virtual covalent bonds. In Figures $3 a-3 c$, the specific heat as well as the fluctuations of gyration radius and end-to-end distance
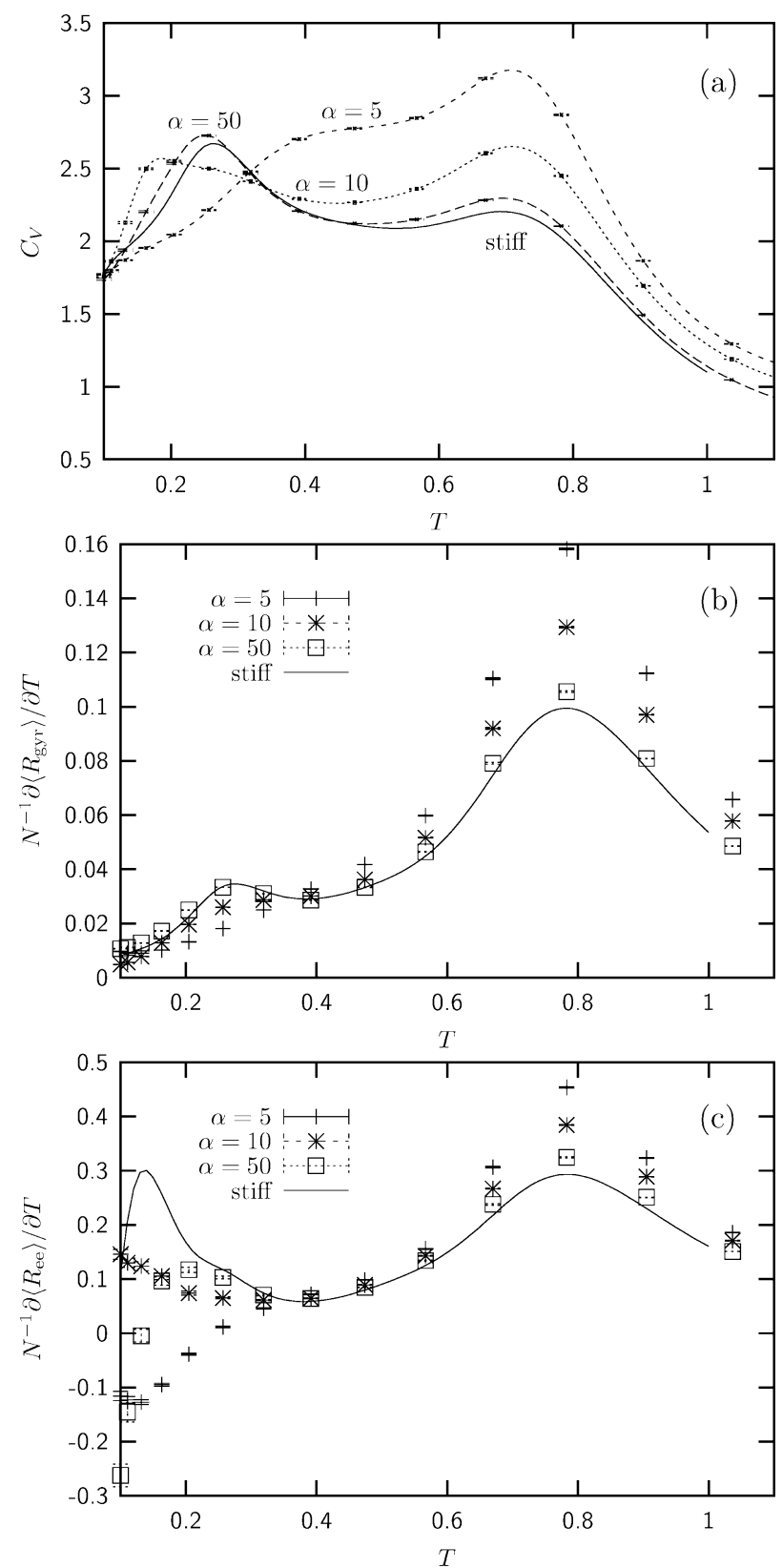

Figure 3. RE-MC results for the heteropolymer S1: (a) specific heat per monomer, fluctuations of (b) gyration radius and (c) end-to-end distance as functions of the temperature for different strengths $\alpha$ of the harmonic bonds. For comparison, also the results for a stiff polymer (fixed bond length) are shown [for $C_{V}$, the effect of the reduced number of degrees of freedom is artificially compensated by a constant offset $(N-1) / 2 N]$. Jackknife error bars are also shown, but are very small. 

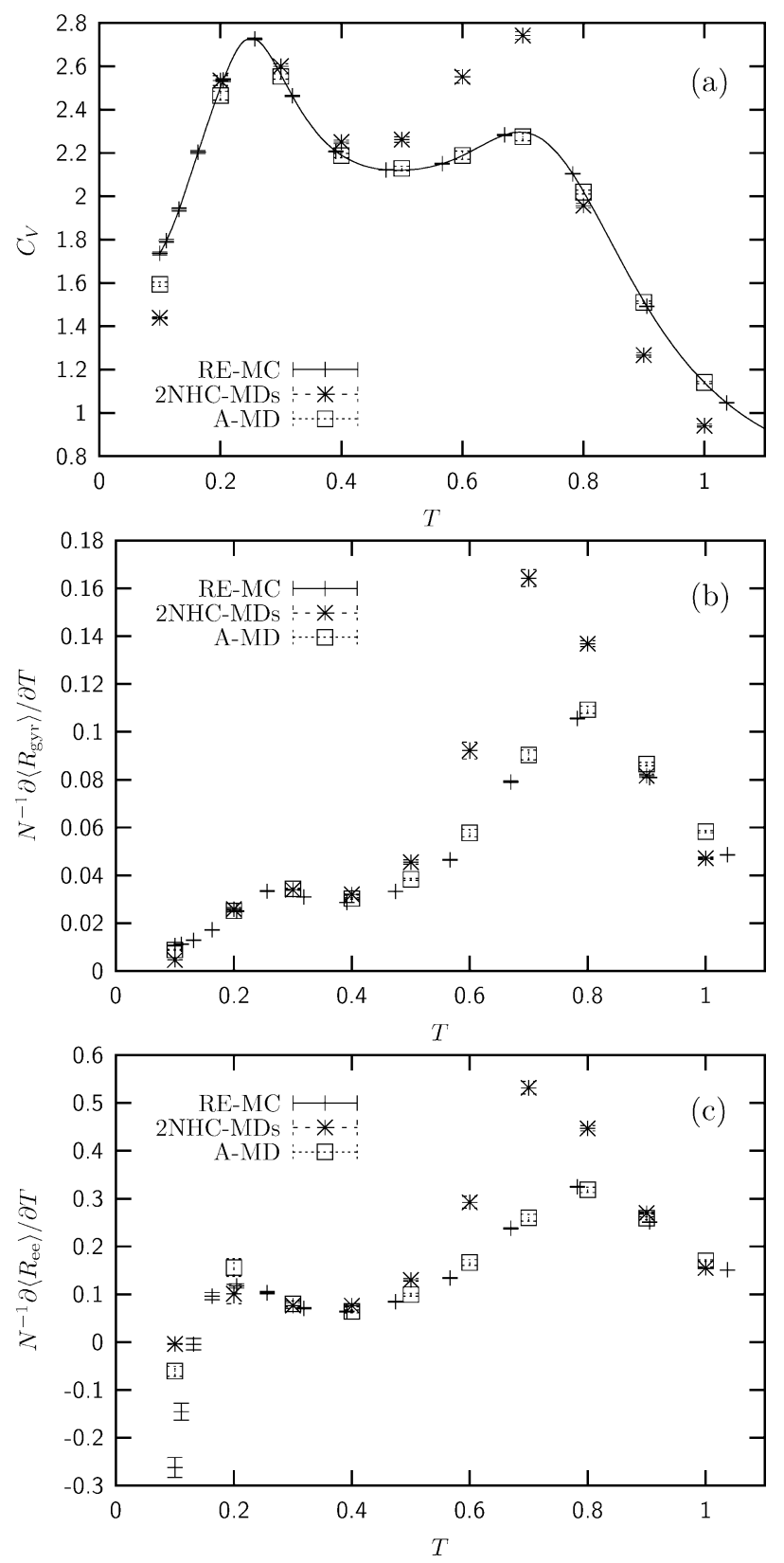

Figure 4. Comparison of results from RE-MC, 2NHC-MDs, and AMD simulations with bond strength $\alpha=50$ for the heteropolymer S1: (a) specific heat per monomer, fluctuations of (b) gyration radius and (c) end-to-end distance as functions of the temperature. Jackknife error bars are of symbol sizes or smaller.

are shown for different choices of the harmonic coupling strengths $\alpha=5,10,50$. These results were obtained from RE-MC simulations with high precision and serve as reliable reference data. For large coupling strengths, these data reproduce former results obtained in studies for heteropolymers with stiff bonds, where other sophisticated MC techniques were employed. ${ }^{17,18}$ Error bars shown were calculated using the jackknife binning method.$^{25}$ In all plots, there is a peak around $T \approx 0.8$. Although the maximum values depend on $\alpha$, the peak temperature does not. In fact, these peaks are indications for the conformational transition between random-coil structures and globular conformations and is, therefore, seen in all fluctuations. There is also a second region of activity at lower temperatures, with a comparatively weak signal in $\partial\left\langle R_{\text {gyr }}\right\rangle / \partial T$. Actually, the heteropolymer does not experience a further collapse, but rather an energetically favorable rearrangement of the monomers. This is an indication for the typical heteropolymer-specific effect of the formation of a compact hydrophobic core. The increasing strength of this signal for stiffer bonds can thus be explained with the larger barrier associated with the monomer rearrangement. This is not surprising as the fluctuation width of the springs decreases, i.e., the $N-1$ bond degrees of freedom are "frozen". This effect is maximal for fixed bonds, ${ }^{17}$ where the heteropolymer possesses only $3 N-(N-1)=2 N+1$ degrees of freedom. It should be noted that, due to the different number of degrees of freedom, the specific heat even for the spring model with extremely stiff, but not fixed, bond lengths $\alpha \rightarrow \infty$ differs from the fixed-bond case by $k_{B} / 2$ per bond. In Figure 3, the curves for the fixed-bond case ${ }^{17}$ are included for comparison (for the associated specific heat including the "ficitious" constant offset per monomer, $(N-1) k_{B} / 2 N$, compensating the frozen-bond constraint).

For comparing thermodynamic quantities, we performed REMC simulations and respective A-MD and 2NHC-MDs for S1 with relatively stiff bonds, $\alpha=50$. The results for the specific heat and the fluctuations of gyration radius and end-to-end distance are shown in Figures $4 a-4 c$, respectively. As can clearly be seen, RE$\mathrm{MC}$ and A-MD results coincide for all quantities for a wide range of temperatures. This is obviously not the case for the 2NHC-MDs data points which deviate for temperatures $T>0.4$ seriously from the RE-MC results. The qualitative thermodynamic behavior, i.e., the occurrence of conformational transitions, is still identified in all cases and the peak temperatures are comparable, but the quantitative agreement for the fluctuating quantities between RE-MC and $2 \mathrm{NHC}-\mathrm{MDs}$ is very poor.

In order to see whether the deviation is due to a too small length of the Nosé-Hoover chain, we repeated the NHC-MD simulations with $M=3$ and $M=4$ thermostats. A noticeable change of the results was, however, not expected as no model-specific additional conserved quantities were identified. The simplicity of the model allowed for extremely long equilibration times and run lengths, as listed in Table 1. Differences between the independent MD runs, worth to be discussed in detail, were not found. This is confirmed by the results shown in Figure 5, where the output of the several tested NHC-MD variants for the specific heat is plotted. In particular, increasing the run time of 2NHC-MDs by a factor of 20 (2NHC-MDl) does not improve the results noticeably.

Another check we performed was to change the virtual masses. From the analysis of the dependence of the statistical results on the masses for the one-dimensional harmonic oscillator in The Choice of the Virtual Masses for the Heat-Bath-Coupling Degrees of Free$d o m$, it is clear that a careful choice of the $Q_{k}$ values is required to obtain reliable results. The simulations leading to the 2NHC-MDs results shown in Figures $4 a-4 c$ and Figure 5 were based on the relation (17) as suggested in Ref. 10. In order to see the influence of the virtual masses on the results, we used in additional simulations different simple rescalings of the $Q_{k}$ values given by eqs. (17) and (19), i.e., for our polymer with $\alpha=50, m=1$, and $N=20$, we chose as reference values $Q_{1}(T)=0.6 T$ and $Q_{2}(T)=0.01 T$. 


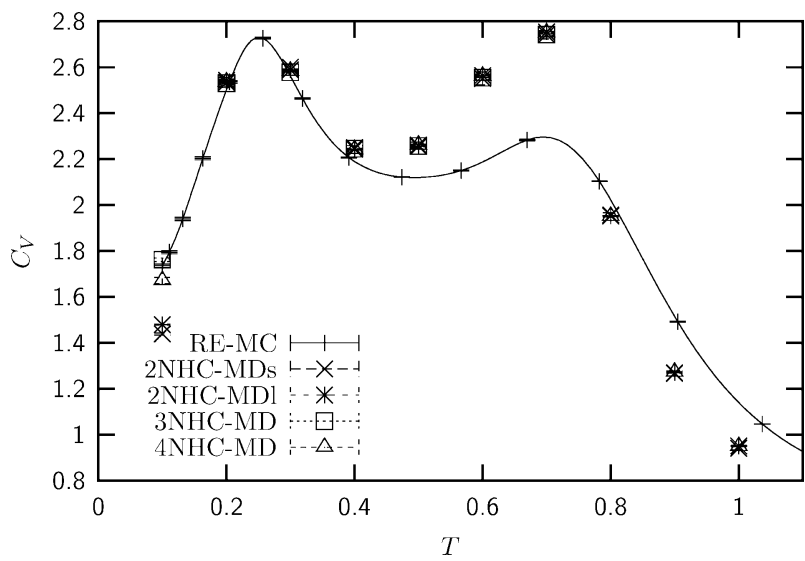

Figure 5. Results for the specific heat as obtained with the variants 2NHC-MDs, 2NHC-MDl, 3NHC-MD, and 4NHC-MD. For comparison, the RE-MC curve is also shown.

The results for the specific-heat estimates are shown in Figure 6, again compared with the replica-exchange MC values. Actually, the results depend on the virtual masses, but simple rescaling obviously does not solve the problem: The "best" choice for high temperatures, $\tilde{Q}_{k}=Q_{k} / 1000$, produces wrong results in the intermediate temperature region. It seems that the temperature dependence of the optimal $Q_{k}$ 's is nontrivial and the assumption of a linear $T$-dependence in eq. (17) is insufficient. If no data are available for comparison, the quality of the 2NHC-MDs results cannot be appraised. This is, of course, a substantial problem.

In further tests, it also turned out that the 2NHC-MDs results depend on the initially chosen conformation, i.e., the initial condition for the Nosé-Hoover dynamics was not forgotten throughout the run. In consequence, the thermodynamic equilibrium state space was not sampled reliably. This aspect is directly connected with the general MD heteropolymer folding problem: Starting from an unfolded, denatured conformation, the native, folded state was rarely found. On the other hand, also unfolding from the initialized native conformation is slow, but the sampling in intermediate and high temperature regions is clearly improved. From the above results, it is not surprising that the strongest deviations, compared with the RE-MC results, are noticed close to the collapse transition, where an appropriate sampling of the collapsed and the random-coil phase is required.

We have also repeated the simulations for a small polymer with anharmonic interactions among non-bonded monomers in order to find out whether systematic deviations of statistical quantities are also present in this much simpler system. The potential energy of this system,

$$
V(\mathbf{R})=v_{\text {harm }}(\mathbf{R})+v_{\text {anharm }}(\mathbf{R}),
$$

consists of the harmonic bond energy $v_{\text {harm }}$ as already defined in eq. (5) and an anharmonic potential $v_{\text {anharm }}$ for the interaction between non-bonded monomers:

$$
v_{\text {anharm }}=\sum_{l=1}^{N-1} \sum_{m=l+1}^{N}\left[\gamma_{1}\left(r_{l m}-b_{0}^{\text {non }}\right)^{2}+\gamma_{2}\left(r_{l m}-b_{0}^{\text {non }}\right)^{4}\right] .
$$

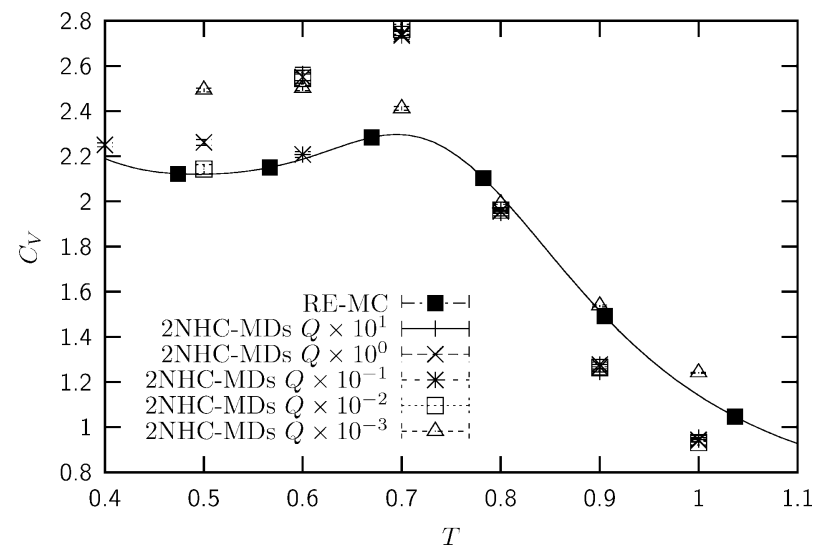

Figure 6. Collapse transition region of the specific heat as obtained from 2NHC-MDs runs for different choices of the virtual masses $Q_{k}$. The reference values $Q=Q_{1,2}$ are given by eq. (17) as suggested in Ref. 10.

In our simulations, the model was parametrized by setting $\alpha=50$, $\gamma_{1}=10$, and $\gamma_{2}=1$. The equilibrium distance between bonded and nonbonded monomers was $b_{0}=b_{0}^{\text {non }}=1$. The simulations were perfomed for a small homopolymer with $N=5$ monomers (sequence $A_{5}$ ). Again, statistics of parallel tempering MC simulations was compared to results from MD simulations with NHC thermostat. The particular parameters and run lengths were chosen according to the values given for RE-MC and 2NHC-MDl in Table 1. Two types of initial conformations were used for the MD simulations. In one set of simulations, we started from random conformations at each temperature, while in the other case low-energy crystalline conformations were chosen, constructed from a tetrahedron with an additional monomer mirrored at one face. In Figure 7 , results for the specific heats of the anharmonic 5-mer, obtained with RE-MC and differently initialized 2NHC-MDl, are shown. Again, the results of the Nosé-Hoover MD simulations deviate systematically from the MC output. As for the Lennard-Jones heteropolymer

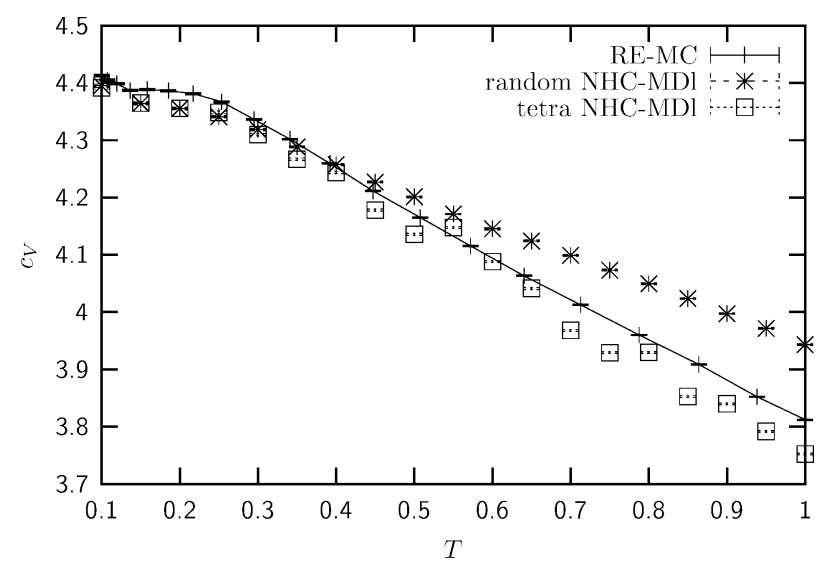

Figure 7. Specific heats obtained from RE-MC and 2NHC-MD1 runs with random and ordered start conformations for a 5-mer with anharmonic interaction (25), (26) between nonbonded monomers. 


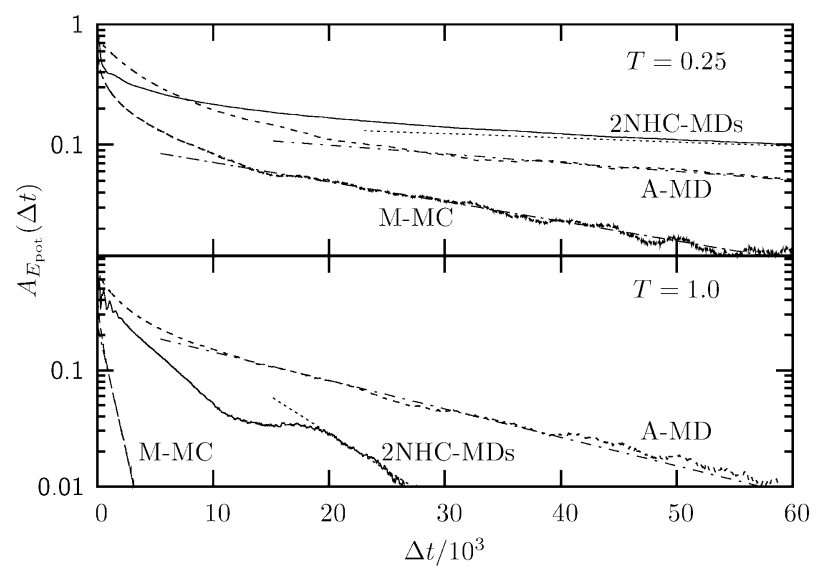

Figure 8. Autocorrelation functions from M-MC, A-MD, and 2NHCMDs runs and fits $\sim \exp \left(-\Delta t / \tau_{\exp }\right)$ at temperatures $T=0.25$ and $T=1.0$.

$\mathrm{S} 1$, the deviations become stronger at high temperatures, where the NHC coupling to the heat-bath is more relevant.

\section{Autocorrelation Time Analysis for MD and MC}

For long time intervals $\Delta t$, the autocorrelation function (18) decays exponentially, $A_{s}(\Delta t) \sim \exp \left(-\Delta t / \tau_{\exp }\right)$, where $\tau_{\exp }$ is the exponential autocorrelation time. Therefore, the autocorrelation time is a measure for the decay rate of correlations in equilibrium and depends on the dynamics of the algorithm which is employed to generate the time series data. The statistical significance of a data set is connected with small temporal correlations. This means that in a time series with $L$ entries only about $n_{\text {eff }} \approx L / \tau_{\text {exp }}$ data points are uncorrelated and determine the statistical error of the data. For this analysis, the origin of the time series, i.e., the inherent algorithmic time scale, is irrelevant and, therefore, the autocorrelation functions of time series obtained with different methods can be compared. In particular, the autocorrelation time is a good quantitative measure for the efficiency of algorithms in sampling the relevant state space.

For two fixed temperatures, $T=0.25$ and $T=1.0$, we have compared the autocorrelation functions and autocorrelation times of standard M-MC simulations, A-MD, and 2NHC-MDs for the Lennard-Jones heteropolymer S1. In the M-MC case, the time difference $\Delta t$ is measured in MC sweeps, where, within a single sweep, the coordinates of all monomers are sequentially tried to be moved randomly. The conformational changes are accepted according to the Metropolis criterion, with the acceptance rate adjusted at around $50 \%$. For the MD runs, $\Delta t$ is measured in units of time steps $\delta t$.

Figure 8 shows the respective autocorrelation functions of the M-MC, A-MD, and 2NHC-MDs simulations for $T=0.25$ and $T=1.0$. In Table 2, we have listed the autocorrelation times for the two fixed temperatures. Similarly to the MC results, the autocorrelation time in the MD runs also decreases with temperature. The autocorrelation times differ noticeably for the three methods compared with each other. Not unexpectedly, autocorrelations decay fastest for M-MC. The values of $\tau_{\text {exp }}$, which are of the order $\mathcal{O}\left(10^{3}-10^{5}\right) \mathrm{MC}$ sweeps respective MD time steps for the two temperatures considered, are much smaller than the run lengths of the simulations (see Table 1 ). Thus, $n_{\text {eff }} \sim 10^{4} \ldots 10^{5}$ data points are uncorrelated in all MC and MD runs. For this reason, the statistical error bars for all of our MC and MD results are very small. Thus, the partly large deviations in the results for energetic and structural fluctuations, in particular near the conformational transitions (see, e.g., Figure 4) and for high temperatures (as in Figure 7), are not of statistical nature. Rather, we conclude that the system behaves non-ergodic in 2NHC-MD i.e., not all sections of the physical phase space being thermodynamically relevant at the given temperature $T$ are covered by intersection points of the trajectory projected from the extended Nosé-Hoover phase space. The almost constant part of the $T=12$ NHC-MD curve in Figure 8 around $\Delta t=10000 \ldots 20000$ is also an indication that the system got stuck in a local free-energy minimum.

\section{Summary}

In this study, we have shown by explicit comparison with results from Monte Carlo simulations that even for a minimalistic model at mesoscopic length scales Nosé-Hoover chain molecular dynamics simulations are not capable to reproduce the correct thermodynamic behavior of heteropolymers. From our analysis, we conclude that for the polymer systems investigated in our study, the proper stable thermodynamic equilibrium cannot be reached in molecular dynamics simulations with Nosé-Hoover chain thermostats and results depend on the initialization of the systems. In consequence, the sampling of folding and unfolding events is insufficient. Although the results for low temperatures are comparable with replica-exchange Monte Carlo data, it should be noted that in the NHC-MD runs folding events were hardly observed. Therefore, the correct formation of the hydrophobic core towards the native fold did not happen and sampling at very low temperatures, i.e., in the hydrophobic-core dominated region, is only reasonable, if the MD run is initialized with the native state. For intermediate and high temperatures, we find a serious dependence of the results on the choice of the values for the virtual masses of the heat-bath coupling degrees of freedom.

The exemplified heteropolymer used in our study possesses only 20 monomers and is thus comparatively small. Its folding characteristics is not particularly complex as the stiffness of the virtual bonds has been relaxed to simplify the MD implementation. It should be noted, however, that substituting the Nosé-Hoover thermostat against the Andersen thermostat with random collisions significantly improves the MD results.

Table 2. Exponential Autocorrelation Times from M-MC, A-MD, and 2NHC-MDs Runs at $T=0.25$ and $T=1.0$.

\begin{tabular}{llr}
\hline & $T$ & \multicolumn{1}{c}{$\tau_{\exp }$} \\
\hline M-MC & 0.25 & $27 \times 10^{3}$ \\
A-MD & 0.25 & $60 \times 10^{3}$ \\
2NHC-MDs & 0.25 & $124 \times 10^{3}$ \\
M-MC & 1.0 & $1 \times 10^{3}$ \\
A-MD & 1.0 & $18 \times 10^{3}$ \\
2NHC-MDs & 1.0 & $7 \times 10^{3}$ \\
\hline
\end{tabular}


In consequence, for statistical analyses of heteropolymers in a wide range of temperatures, the applicability of canonical constanttemperature molecular dynamics simulations with deterministic thermostats is rather limited. For realistic models, the complexity of the microscopic description at the atomic scale is known to extremely slow down NHC-MD folding simulations. Here, however, we used a much simpler coarse-grained model and folding events have also not been adequately recovered.

It should be noted, however, that NHC-MD has proven to be quite successful in explaining dynamic processes at time scales much shorter than folding times, where, e.g., selected biological functions of proteins under physiological conditions can be studied. Interesting examples, where the application of NHC-MD methods proved very useful, are water penetration into a cell through the aquaporin membrane protein ${ }^{26}$ and the ATP synthase, where the catalytic subunits of F1, embedded into the membrane F0 proton channel, partially act as rotating "molecular motor" that promotes dehydration of ADP and P to ATP. ${ }^{27}$ Such studies require that the native folds of the proteins must be known as these are used as input. Substantial conformational changes of the proteins do not occur or are limited to small segments of a few amino acids.

\section{References}

1. Allen, M. P.; Tildesley, D. J. Computer Simulation of Liquids; Clarendon Press: Oxford, New York, 1987.

2. Frenkel, D.; Smit, B. Understanding Molecular Simulation; Academic: San Diego, 2002.

3. Landau, D.; Binder, K. A Guide to Monte Carlo Simulations in Statistical Physics, 2nd ed., Cambridge University Press: Cambridge, 2005.

4. Berg, B. A. Markov Chain Monte Carlo Simulations and Their Statistical Analysis; World Scientific: Singapore, 2004.

5. Koch, W.; Holthausen, M. C. A Chemist's Guide to Density Functional Theory, 2nd ed; Wiley: Weinheim, 2001.
6. Gross, D. H. E. Microcanonical Thermodynamics; World Scientific: Singapore, 2001.

7. Andersen, H. C. J Chem Phys 1980, 72, 2384.

8. (a) Nosé, S. J Chem Phys 1984, 81, 511; (b) Nosé, S. Mol Phys 1984, 52, 255; (c) Nosé, S. 1986, 57, 187; (d) Nosé, S. 2002, 100, 191.

9. (a) Hoover, W. G. Phys Rev A 1985, 31, 1695; (b) Hoover, W. G. 1986, 34, 2499.

10. Martyna, G. J.; Klein, M. L. J Chem Phys 1992, 97, 2635.

11. Pierleoni, C.; Ryckaert, J.-P. Mol Phys 1992, 75, 731.

12. Bennemann, C.; Paul, W.; Binder, K.; Dünweg, B. Phys Rev E 1998, 57,843 .

13. Hukushima, K.; Nemoto, K. Phys Soc J 1996, 65, 1604.

14. (a) Stillinger, F. H.; Head-Gordon, T.; Hirshfeld, C. L. Phys Rev E 1993, 48, 1469; (b) Stillinger, F. H.; Head-Gordon, T. Phys Rev E 1995, 52, 2872.

15. Ryckaert, J. P.; Ciccotti, G.; Berendsen, H. J. C. J Comput Phys 1977, 23, 327.

16. Andersen, H. C. J Comput Phys 1983, 52, 24.

17. Bachmann, M.; Arkın, H.; Janke, W. Phys Rev E 2005, 71, 031906.

18. (a) Schnabel, S.; Bachmann, M.; Janke, W. Phys Rev Lett 2006, 98, 048103; (b) Schnabel, S.; Bachmann, M.; Janke, W. J Chem Phys 2007, 126, 105102.

19. Junghans, C.; Bachmann, M.; Janke, W. Phys Rev Lett 2006, 97, 218103.

20. Verlet, L. Phys Rev 1967, 159, 98.

21. Martyna, G. J.; Tuckerman, M. E.; Tobias, D. J.; Klein, M. L. Mol Phys 1996, 87, 1117.

22. Yoshida, H. Phys Lett A 1990, 150, 262.

23. Suzuki, M. J Math Phys 1991, 32, 400.

24. (a) Bachmann, M.; Janke, W. Phys Rev Lett 2003, 91, 208105; (b) Bachmann, M.; Janke, W. J Chem Phys 2004, 120, 6779.

25. (a) Miller, R. G. Biometrika 1974, 61, 1; (b) Efron, B. The Jackknife, the Bootstrap, and Other Resampling Plans; Society for Industrial and Applied Mathematics [SIAM]: Philadelphia, 1982.

26. de Groot, B. L.; Frigato, T.; Helms, V.; Grubmüller, H. J Mol Biol 2003, 333, 279.

27. Böckmann, R. A.; Grubmüller, H. Biophys J 2003, 85, 1482. 